\title{
Application of the lattice Boltzmann method to transition in oscillatory channel flow.
}

\author{
J. A. Cosgrove, J. M. Buick, S. J. Tonge, C. G. Munro, \\ C. A. Greated and D. M. Campbell \\ Department of Physics and Astronomy, The University of Edinburgh, Edinburgh \\ EH9 3JZ.
}

\begin{abstract}
In this study the applicability of the lattice Boltzmann method to oscillatory channel flow with a zero mean velocity has been evaluated. The model has been compared to exact analytical solutions in the laminar case $\left(R e_{\delta}<100\right.$, where $R e_{\delta}$ is the Reynolds number based on the Stokes layer) for the Womersley parameter $1<\alpha<31$. In this regime, there was good agreement between numerical and exact analytical solutions. The model was then applied to study the primary instability of oscillatory channel flow with a zero mean velocity. For these transitionary flows the parameters were varied in the range $400<R e_{\delta}<1000$ and $4<\alpha<16$. Disturbances superimposed on the numerical solution triggered the two-dimensional primary instability. This phenomenon has not been numerically evaluated over the range of $\alpha$ or $R e_{\delta}$ currently investigated. The results are consistent with quasi-steady linear stability theories and previous numerical investigations.
\end{abstract}

PACS numbers: 47.11.+j, 47.15.-x, 47.27.Cn.

Submitted to: J. Phys. A: Math. Gen. 


\section{Introduction}

This investigation is concerned with the lattice Boltzmann method (LBM) applied to the generation of the primary instability in oscillatory flow. Oscillatory and pulsating flows occur both naturally and in engineering applications ranging from chemical mixing and offshore engineering to respiratory, vascular and acoustic flows. Here, purely oscillatory flow with a zero-mean velocity in wall-bounded channels is examined. This problem was chosen to evaluate the LBM applied to unsteady flow as it is one of the simplest unsteady flows possible, has an exact analytical solution of the Navier-Stokes equations and can serve as a prototype problem from which conclusions can be inferred for more complicated non-periodic flows.

For the laminar regime of such flows, experimental and analytical results agree well and so the LBM is compared to an exact solution enabling the accuracy of the implemented model to be examined. In the transition regimes there are conflicting results between experimental measurements $[1,2,3,4,5,6]$ and analytical stability analyses $[7,8]$ in terms of predicting the phase of the onset of turbulence, with some stability analyses not predicting instability growth over a large range of wavenumber and Reynolds number (based on Stokes length) [9]. Evidently numerical modelling plays an important role in studying such phenomena although there have been relatively few numerical studies performed relating to purely oscillatory flows in the transition regime, most notably [10].

The relative merits of the LBM itself are now well established, [11, 12]; it lends itself naturally to parallel implementation, boundary conditions are easily applied and it is easily interpreted in physical terms. Historically, the LBM has evolved from lattice gas models. Lattice gas models belong to a class of cellular automata consisting of a lattice of sites which can take on a finite number of states. The automaton evolves in discrete time steps with the state at each site at any time determined by its own state and the state of a set of neighbouring sites at the previous time step. The lattice gas model represents fluid particles colliding and propagating throughout a fluid and hence the ease of physical interpretation. Importantly, the LBM overcomes difficulties associated with the lattice gas models by considering the evolution of distribution functions rather than individual fluid particles.

\section{Oscillatory Flow}

The dominant features of oscillatory flows, in the context of the current investigation, are characterised by two non-dimensional parameters. The first is the Womersley parameter $\alpha=a \sqrt{\omega / \nu}$ with $a$ the channel half-width, $\omega$ the angular frequency and $\nu$ the kinematic viscosity of the fluid. The second is the Reynolds number based on the Stokes layer thickness, $R e_{\delta}=U_{0} \delta / \nu$ with $U_{0}$ the maximum amplitude of the velocity and $\delta=\sqrt{2 \nu / \omega}$ is the Stokes layer thickness. The Stokes parameter $\Lambda$ is often used instead of $\alpha$ with $\Lambda=a / \delta=\alpha / \sqrt{2}$. 
Previous experimental studies of such flows $[1,2,3,4,5,6]$ indicate that there are three general categories of flow regimes; laminar flow, disturbed laminar flow, and intermittently turbulent flow. The laminar regime and the development of the primary instability which is an important mechanism in the transition to intermittent turbulent flow are of interest in this investigation. For an analytical solution corresponding to the laminar case [13], consider flow in an infinitely long, two-dimensional channel of width $2 a$ which is being driven by a sinusoidally varying pressure gradient

$$
\frac{\partial p}{\partial x}=-p^{*} e^{i \omega t}
$$

where the $x$-axis is in the centre of the channel parallel to the walls, the streamwise direction, and the $y$-axis is perpendicular to the walls, the spanwise direction. Here $p^{*}$ is the maximum amplitude of the sinusoidally varying pressure gradient. Looking for a solution in which the velocity is independent of the $x$-position, it is evident from the continuity equation, that $u_{y}$ takes its boundary value everywhere. Thus the NavierStokes equation reduces to

$$
\frac{\partial u_{x}}{\partial t}=-\frac{1}{\rho} \frac{\partial p}{\partial x}+\nu \frac{\partial^{2} u_{x}}{\partial y^{2}},
$$

with boundary conditions $\left.u_{x}\right|_{y= \pm a}=0$ and where $\rho$ is the fluid density. Substituting the expression for the pressure gradient, equation (1), into equation (2) and writing the velocity as

$$
u_{x}=u(y) e^{i \omega t}
$$

leads to the final form of the equation: $\partial^{2} u / \partial y^{2}-(i \omega u) / \nu=-p^{*} / \rho \nu$. The final expression for the velocity is [13]

$$
u_{x}=\Re\left\{\frac{p^{*}}{i \omega \rho}\left[1-\frac{\cosh \left[\frac{1}{\sqrt{2}}(\alpha+i \alpha) \frac{y}{a}\right]}{\cosh \left[\frac{1}{\sqrt{2}}(\alpha+i \alpha)\right]}\right] e^{i \omega t}\right\},
$$

where $\Re$ denotes the real part of the solution. Noting that $\cosh (\alpha+i \alpha) \simeq 1+i \alpha^{2}$, for small $\alpha$, Equation (4) reduces to

$$
u_{x} \simeq \frac{p^{*}}{2 \nu \rho}\left(a^{2}-y^{2}\right) \cos (\omega t) .
$$

which is simply a quasi-steady flow in which the velocity profile at any time is the same as that for Poiseuille flow with the relevant forcing term. For large $\alpha$ we can express $\cosh (\alpha+i \alpha) \simeq \frac{1}{2} e^{|\alpha|} e^{i|\alpha|}$ in which case Equation (4) reduces to

$$
u_{x} \simeq \frac{p^{*}}{\omega \rho} \sin (\omega t)
$$

that is, a flat profile across the tube which is oscillating sinusoidally with time.

The second category of disturbed laminar flow refers to observations of small amplitude perturbations superimposed on the velocity traces during the acceleration phase of the flow before reverting to the laminar case. The transition of flows to this category seems to be quite sensitive to the particular experimental set-up, whereas the 
transition to category three, where explosive turbulent bursts are observed towards the end of the acceleration phase, is much more clearly defined with transition occurring in the range $500<R e_{\delta}<550$. These turbulent bursts are largely independent of the experimental set-up and there is consistent published data available $[1,2,3,4,5,6]$. Despite this, the number of successful theoretical investigations is limited since there is a lack of derivations of the critical Reynolds number based on linear theories from which non-linear theories can be developed. It is generally agreed that transition to turbulence is due to local instabilities in the Stokes boundary layer as long as the boundary layer is small compared to the physical dimensions of the problem. In view of the general consensus on the location of the onset of turbulence much of the theoretical work revolves around studying infinitesimal disturbances in the Stokes layer for which the equations of motion can be linearized. The problem is generally approached using Floquet or quasi-steady theories $[7,8,9]$. In quasi-steady theories the spatially dependent profiles of the flow are examined at different instances in time and therefore the profiles have only a parametric dependence on time. This method differs from time dependent ones that use Floquet theory to examine disturbances in the periodic steady state. However these either do not predict the correct phase for the onset of turbulence or do not predict instability growth over a large range of $R e_{\delta}$ and wavenumber.

The discord between theory and experiment can be explained by a secondary instability mechanism whereby the existence of finite-amplitude waves leads to vortical structures within the flow that are inviscidly unstable to three dimensional perturbations. The broadband nature of this instability provides a mechanism for the rapid generation and growth of small scales from a smooth velocity profile. Akhavan et al. [10] has performed a numerical simulation based on this mechanism which is consistent with experimental observations.

The transition to turbulence in many shear flows, such as pipe, Poiseuille, Couette, free shear layer and boundary-layer flows, are the result of instabilities of two-dimensional equilibrium waves to three-dimensional disturbances. Here we are concerned with the primary instability which generates the initial two dimensional disturbance. The range of $\alpha$ or $R e_{\delta}$ currently investigated provides a consistent and broad evaluation of the phenomenon. In the current implementation an excitation of all simulated wavenumbers is used as a certain level of noise is always present throughout the flow field [10]. This differs from previous theoretical and numerical studies which have largely concentrated on examining growth (or decay) rates of initial perturbations at critical phases within the cycle and in the case of numerical studies of a single wavenumber. Furthermore in the case of Akhavan et al. [10], a linearised model of the Navier-Stokes equation is used. Prior to discussing the results of the simulations, some theoretical and implementation aspects of the LBM are given. 


\section{The lattice Boltzmann model}

In the current investigation the LBM is implemented in two dimensions on a square lattice, using the nine vector model [14], the so-called D2Q9 lattice. The discrete lattice Boltzmann equation for the particle distribution function takes on a similar form to the kinetic equation of the lattice gas model

$$
f_{i}\left(\mathbf{x}+\mathbf{e}_{i}, t+1\right)-f_{i}(\mathbf{x}, t)=\Omega_{i}(f(\mathbf{x}, t))
$$

where the distribution functions $f_{i}(\mathbf{r}, t)$ evolve on a fixed lattice along the links, $\mathbf{e}_{i}$, where $i$ is an integer labelling the link and

$$
e_{i}= \begin{cases}(0,0) & i=0 \\ (\cos [(i-1) \pi / 2], \sin [(i-1) \pi / 2]) & i=1,2,3,4 \\ (\cos [(i-5) \pi / 2+\pi / 4], \sin [(i-5) \pi / 2+\pi / 4]) & i=5,6,7,8\end{cases}
$$

The fluid density, $\rho$, and velocity, $\boldsymbol{u}$, at each site can be calculated from the distribution functions as

$$
\rho=\sum_{i} f_{i} \quad \text { and } \quad \rho u_{\alpha}=\sum_{i} f_{i} e_{i \alpha}
$$

where the Greek subscripts represent vector components and summation over repeated Greek indices is assumed. Using the Bhatnagar-Gross-Krook approximation [15, 16] to the collision operator greatly simplifies the Boltzmann model with the approximation given by

$$
\Omega_{i}=\frac{1}{\tau}\left[f_{i}(\mathbf{x}, t)-\overline{f_{i}}(\mathbf{x}, t)\right]
$$

where $\tau$ is the relaxation time and $\overline{f_{i}}$ is the equilibrium value of the distribution function. This takes the general form [11],

$$
\bar{f}_{i}(\boldsymbol{r}, t)=\rho\left(a_{i}+b_{i} \boldsymbol{e}_{i} \cdot \boldsymbol{u}+c_{i}\left(\boldsymbol{e}_{i} \cdot \boldsymbol{u}\right)^{2}+d_{i} u^{2}\right), \quad i=0, . ., 8
$$

where $a_{i}, b_{i}, c_{i}$ and $d_{i}$ are constants that depend on the specific lattice being used and the required properties of the fluid. The constants $a_{i}, b_{i}, \ldots, d_{i}$ are chosen so that the collisions satisfy the conservation of mass and momentum and that the fluid is isotropic, exhibits Galilean invariance and satisfies the continuity and Navier-Stokes equations. The values applied here are [14]

$$
a_{i}=w_{i}, \quad b_{i}=3 w_{i}, \quad c_{i}=\frac{9}{2} w_{i}, \quad \text { and } \quad d_{i}=-\frac{3}{2} w_{i},
$$

where $w_{0}=4 / 9, w_{1}=w_{2}=w_{3}=w_{4}=1 / 9$ and $w_{5}=w_{6}=w_{7}=w_{8}=1 / 36$. The distribution function can be expanded about the equilibrium value, $f_{i} \rightarrow \bar{f}_{i}+$ $\epsilon f_{i}^{(1)}+\epsilon^{2} f_{i}^{(2)}$, where $\epsilon$ is the Knudsen number which must be small. By substituting this expansion into Equation (7), performing a Taylor expansion and after some algebraic manipulation the conservation of mass equation,

$$
\frac{\partial \rho}{\partial t}+\frac{\partial \rho u_{\alpha}}{\partial x_{\alpha}}=0
$$


can be derived. Similarly a momentum equation can be found as

$$
\frac{\partial \rho u_{\alpha}}{\partial t}+\frac{\partial \rho u_{\beta} u_{\alpha}}{\partial x_{\beta}}=-\frac{\partial p}{\partial x_{\alpha}}+\nu \frac{\partial}{\partial x_{\beta}}\left(\rho \frac{\partial u_{\beta}}{\partial x_{\alpha}}+\rho \frac{\partial u_{\alpha}}{\partial x_{\beta}}\right) .
$$

When the flow speed is sufficiently smaller than the speed of sound, $c_{s}=1 / \sqrt{3}$ in lattice units, Equations (13) and (14) represent the Navier-Stokes equation for an incompressible fluid in two-dimensions with equation of state $p=\rho c_{s}^{2}$ and kinematic viscosity $\nu=(2 \tau-1) / 6[17]$.

A number of schemes have been proposed to implement a no-slip boundary condition, see for example $[18,19,20]$. At the boundary there are a number of distribution functions which are not defined after the streaming action of the LBM implementation. These are in the directions pointing away from the boundary. When applying the boundary conditions it is necessary to determine values for these distribution functions. The simplest method is a bounce-back boundary which is derived from the lattice gas model in which any particle approaching the boundary is reflected back in the direction from which it came. An alternative approach proposed by Noble et al. [18] can be applied to a plane wall parallel to one of the link vectors $\mathbf{e}_{i}$. In this case there are three unknown distribution functions pointing from the solid boundary into the fluid. These three unknowns are found such that $\sum_{i} f_{i} e_{i \alpha}=\tilde{u}_{\alpha}$, where $\tilde{\mathbf{u}}$ is the known wall velocity (zero velocity for a stationary no-slip boundary) and the internal energy of the system is conserved during the collision. Here both systems of boundary conditions were implemented; however, the simple bounce back condition provided adequate accuracy and was therefore applied in all the simulations presented here. When then bounce back boundary condition is applied at a halfway wall, that is a wall placed halfway between a row of "boundary sites" and a row of "fluid sites", the boundary condition exhibits second-order accuracy $[21,22]$. The bounce back condition is especially useful when considering more complex wall geometries. Periodic boundary conditions were imposed at the open ends of the channel.

The oscillating pressure variation in Equation (1) was implemented as a body force $\mathbf{F}=\left(p^{*} \cos \omega t, 0\right)[23]$. This was done by introducing an additional term to the Boltzmann equation [24], Equation (7) to give

$$
f_{i}\left(\mathbf{x}+\mathbf{e}_{i}, t+1\right)-f_{i}(\mathbf{x}, t)=\Omega_{i}(f(\mathbf{x}, t))+3 \frac{2 \tau-1}{2 \tau} w_{i} F_{\alpha} e_{i \alpha}
$$

and re-defining the fluid velocity [24] (Equation( 9)) as

$$
\rho u_{\alpha}=\sum_{i} f_{i} e_{i \alpha}+\frac{F_{\alpha}}{2} .
$$

This implementation of a body force can be shown to satisfy the continuity and NavierStokes equations up to second order [24].

In the laminar simulations, the velocities are the same at all points in the streamwise direction so a channel length of 5 sites was used to improve computational efficiency. In the turbulent simulations the length of the channel was varied in the range 
$2 N<M<10 N$ for selected values of $\alpha$ and $R e_{\delta}$. Here $N$ is the grid size in the streamwise direction. No significant difference were found in the results between the differing channel lengths in the context of the present work and so a channel length of $M \approx 2 N$ was chosen for computational efficiency. Since a good agreement is observed between the simulations and the analytic theory in the laminar case and since the simulation can take a significant number of oscillations to converge from an initially stationary state the turbulent simulations were initialised with a constant density and with a velocity calculated from Equation (4).

For the Reynolds numbers considered in this study the turbulent motion can be simulated directly using the LBM [25, 26]. Martinez et al. [25] use the direct LBM approach to simulate turbulent flows with Reynolds numbers, Re, as high as 10,000 and find good agreement between the LBM results and results obtained using a spectral method. To trigger the turbulence it is necessary to introduce a small random variation into the simulation in order to disturb the inherent symmetry. This was done by perturbing the forcing term to produce small irregularities in the flow.

\section{Results and Discussion}

\subsection{The laminar case}

Figures 1(a) to (d) compare the exact analytical solutions of Equation (4) to numerically evaluated velocity profiles across the channel for $\alpha=1.085,5.606$ and 30.706 . The velocity is normalised by the term

$$
u_{c}=\frac{p^{*} \alpha^{2}}{2 \omega \rho},
$$

which is the velocity that would be observed at the centre of the channel if a constant forcing term per unit volume of $p^{*}$ were applied in the limit of $\alpha \rightarrow 0$. The $y$-axis is non-dimensionalised by dividing by the half-width of the channel as indicated in Figures 1(a) to (d). The first two graphs (Figures 1(a) and (b)) indicate the evolution of an oscillation over an entire period at discrete phases $\omega t=n \pi / 8$ of the driving force, with $n$ an integer, $n=0 \ldots 15$ for $\alpha=5.606$. Figures $1(\mathrm{c})$ and $(\mathrm{d})$ show the evolution of an oscillation with the velocity increasing over half a period for $\alpha=1.0856$ and 30.706 respectively.

Figure 1. Profiles of increasing (growing) (a) and decreasing (b) velocities along the width of a channel for $\alpha=5.606$. Profiles of growing velocity for $\alpha=1.0856$ (c) and $\alpha=30.706$ (d). The phase $\omega t$ indicates the temporal location of the velocity relative to the forcing pressure gradient and is given by $\omega t=n \pi / 8$, where $n$ is an integer, $n=0 \ldots 15$.

Figures 1(c) and (d) indicate respectively that for low and high $\alpha$ values the velocity profiles approach those limits described by Equations (5) and (6). At intermediate 
values of $\alpha$, velocity profiles are obtained with characteristic points of inflexion as shown in Figures 1(a) and (b). These inflexion points are important in the generation and transfer of turbulence and these are discussed more fully in the following sub-section. It is evident from the graphs of Figure 1 that the phase is spatially dependent and that the phase shift between the forcing term and velocity varies as the flow changes from a viscous dominated system at relatively low values of $\alpha$ to a momentum dominated system at relatively high values of $\alpha$, in the laminar regime.

For a quantitative analysis of the errors a global error, $\xi$, was defined as

$$
\xi=\frac{\sum_{t} \sum_{\mathbf{x}}\left|\mathbf{u}_{b}(\mathbf{x}, t)-\mathbf{u}_{a}(\mathbf{x}, t)\right|}{\sum_{t} \sum_{\mathbf{x}}\left|\mathbf{u}_{a}(\mathbf{x}, t)\right|},
$$

where $|f(x)|$ denotes the modulus of $f(x)$. Here the difference between the velocities of the exact analytical solution, denoted by the subscript $a$, and the Boltzmann solution, denoted by the subscript $b$, were calculated and then summed over all points on the discrete lattice and over an entire period of oscillation after a convergence criterion $\zeta$,

$$
\zeta=\sum_{\mathbf{x}} \frac{\left|\mathbf{u}_{b}(\mathbf{x}, t+T)-\mathbf{u}_{b}(\mathbf{x}, t)\right|}{\left|\mathbf{u}_{b}(\mathbf{x}, t+T)\right|} \leq 10^{-7}
$$

was attained. Figure (2) graphs the global errors as a function of the channel width $M$ for a number of different values of $\alpha$. For a given $\alpha$, as the lattice size was increased $\alpha$ was kept constant by varying the period $T$ accordingly within the range $T>10^{3}$; the value of $\tau$ was kept constant. In Figure 2, the straight line has a slope $m=-2$ indicating that, for $T>10^{3}$, the LBM incorporating the halfway bounce back boundary conditions and the second-order accurate forcing term, is itself second order in accuracy. For $T<10^{3}$ errors become significant and are of the order $T^{-1}$, consistent with [27] where oscillatory flow has been investigated for $20<T<500$. He and Luo [28] report a scheme accurate to second order in time however in the present investigation since $T$ is of order $10^{-6}$ for the transition simulations of the following section the temporal error of the current implementation is negligible.

Figure 2. The global error $\xi$ plotted as a function of the channel width $M$ for $\alpha=2.00,9.71$ and 14.00 . The solid curve is a line with slope $m=-2$.

To determine the compressibility effects, the Mach number, $M c$, was varied over the range $9.432 \times 10^{-4}$ to $3.772 \times 10^{-1}$ by varying the pressure gradient term from $2 \times 10^{-6}$ to $8 \times 10^{-4}$. The period, viscosity and grid size were kept constant, $T=1600, \nu=0.066$ and $M=80$ giving $\alpha=9.708$. In all cases the global error was found to be constant to within $\pm 5 \times 10^{-15}$ with the difference between results within an order of magnitude of the numerical rounding error. Therefore the implemented model is effectively incompressible in the laminar regime. We note that with reference to the turbulence regime mentioned in the following section, the fluctuating velocities at the onset of turbulence have $M c<10^{-3}$ with the maximum fluctuating velocities having $M c$ of order $10^{-2}$. Errors due to compressibility scale as $O\left(M c^{2}\right)[29,25]$ and the value of $M c$ for the fluctuating velocities is significantly smaller than the value of 0.069 used by Martinez et al. 
[25] who obtained a good agreement between LBM and spectral simulations of decaying turbulence. Reider et al. [29] considered the errors for the evolution of a decaying Taylor vortex and showed that the error decreases with increasing spacial resolution until it reaches a saturation point at which the error remains constant. The value of the error at the saturation point is seen to decrease as $O\left(M c^{2}\right)$. The lowest Mach number considered by Reider et al. [29] is 0.03125 which gives a velocity error due to compressibility errors of 0.00057 . The above considerations indicate that the maximum relative velocity error associated with compressibility in the turbulent simulations is negligible.

\subsection{The turbulent regime}

The numerical solutions of the LBM were perturbed to trigger transition. A continual disturbance was superimposed on the flow with all wavenumbers, $\lambda=\left(\lambda_{m}, \lambda_{n}\right)$ in the range $0<\lambda_{m}<2 \pi / M, 0<\lambda_{n}<2 \pi / N$ excited equally, where $M$ and $N$ are the number of grid points in the streamwise and spanwise directions respectively. The disturbance had an energy of order $10^{-2}$, when normalised to the energy of the mean flow is similar in magnitude to those of Akhavan et al. [10]. This resulted in a velocity $\mathbf{U}$ of the form, $\mathbf{U}=\overline{\mathbf{u}}+\mathbf{u}^{\prime}$, with $\overline{\mathbf{u}}$ and $\mathbf{u}^{\prime}$ the mean and fluctuating components respectively. The averaged velocity $\overline{\mathbf{u}}$ was defined as

$$
\overline{\mathbf{u}}(n, \omega t)=\frac{1}{M} \sum_{m=1}^{M} \mathbf{u}(m, n, \omega t) .
$$

For selected values of $\alpha, \overline{\mathbf{u}}$ was also averaged over $P$ oscillations, that is

$$
\overline{\mathbf{u}^{*}}(n, \omega t)=\frac{1}{P} \sum_{p=0}^{P-1} \overline{\mathbf{u}}(n, \omega t+2 \pi p) .
$$

however $\overline{\mathbf{u}^{*}}$ and $\overline{\mathbf{u}}$ did not vary significantly in the context of the current discussion. Furthermore, in the present investigation, $|\overline{\mathbf{u}}|>>\left|\mathbf{u}^{\prime}\right|$ and it was noted that calculating the fluctuating component either as $\mathbf{u}^{\prime}=\mathbf{U}-\overline{\mathbf{u}}$ or alternatively as $\mathbf{u}^{\prime}=\mathbf{U}-\mathbf{u}_{\mathbf{a}}$, where $\mathbf{u}_{\mathbf{a}}=\left\{u_{x}(y, t), u_{y}=0\right\}$ is given by the exact analytical solution of Equation (4), yielded results whose differences were negligible for the purposes of the present discussion. Equation (20) was chosen as the most efficient implementation of the average.

Figure 3. Vector map of the fluctuating velocity after flow reversal showing vortex interaction for $\alpha=14.1, R e_{\delta}=800$

Figure (3) shows the fluctuating component of the velocity for $\alpha=14.1, R e_{\delta}=800$, over a section of the channel. Prior to transition the fluctuating component, due to the numeric perturbation, is several orders of magnitude smaller than that after transition and there are no clearly defined vortex structures such as those in Figure (3). Transition is thus very clearly observed. Similar vortex structures were found for all $\alpha$ and $R e_{\delta}$ 
after the onset of turbulence. Although the vortices interacted in a complex fashion depending, as expected, on $\alpha, R e_{\delta}$ and the phase $\omega t$ of the flow, the following general observations were made. The vortices originated in the Stokes layer at flow reversal, typically growing and shearing to a size of order $\delta$. In some cases several vortices would form and interact, exchanging momentum under this shearing action. These vortices close to the boundary set up counter rotating vortices in the main flow which grew to a size of the order of $a$. The large rotations tended to sweep the turbulence, generated close to the boundary, into the main flow where relatively complex interactions between the small and large eddies and the main flow took place.

These observations can be presented in a more global and quantitative manner by looking at the turbulent intensity $q=\sqrt{ }\left({\overline{u_{m}^{\prime}}}^{2}+{\overline{u_{n}^{\prime}}}^{2}\right)$, with $\overline{u_{m}^{\prime}}$ and $\overline{u_{n}^{\prime}}$ the normalised values of the fluctuating components in the streamwise and spanwise directions respectively. These profiles are rendered as grey-scale plots of intensity with suitably non-dimensionalised variables $q=q(y / a, \omega t)$ for selected values of $\alpha$ and $R e_{\delta}$ in figure 4.2. Superimposed on these plots are lines of zero velocity which serve as references

Figure 4. Grey-scale maps of turbulent intensity plotted as a function of $\omega t$ and non-dimensional channel width, (a) $\alpha=4.5, R e_{\delta}=850$, (b) $\alpha=7.0, R e_{\delta}=850$, (c) $\alpha=14.1, \operatorname{Re}_{\delta}=800$ (d) $\alpha=14.1, \operatorname{Re}_{\delta}=500$. Also shown are lines (dashed) of zero velocity which serve as references of flow reversal as well as solid lines which indicate the temporal evolution of inflexion points.

of flow reversal. Lines which correspond to the temporal evolution of these inflexion points are displayed also. Both these lines were calculated from the exact analytical solutions of Equation (4). For all $\alpha$ and $R e_{\delta}$ investigated, the primary instability is triggered in $(\omega t, y / 2 a)$ space where flow reversal occurs in the region of a point of inflexion or a critical layer which can clearly be seen in Figure 4.2 (a)-(d). This is in agreement with quasi-steady linear stability theories where the least stable eigenmodes have peak energies in the Stokes layer in the vicinity of inflexion points or critical layers and occur at the beginning of the acceleration phase. In Figure 4.2 (a), for $\alpha=4.5$, $R e_{\delta}=850$, transition occurs close to the boundary and this is swept rapidly into the centre of the channel. The turbulence intensity then decreases by almost an order of magnitude. At $\omega t \approx 3.5(1.1 \pi)$ there is a growth in the turbulence in the region of an inflexion point, $y / a=0.4$, and this propagates into the channel down to $y / a \approx-0.5$. The turbulence intensifies and moves towards the centre of the channel over $1 / 10$ of an oscillation before decaying. As $\alpha$ is increased, Figure 4.2 (b), the turbulence evolves in a similar fashion with the intensity increasing. The results also suggest that the instability modes follow the inflexion points as they move away from the wall. Hence the most intense turbulence does not reach the centre of the channel for higher values of $\alpha$, as can be seen in Figure 4.2 (c). Although Kerczek and Davis [9] concluded that the instability modes were unable to take advantage of the Rayleigh mechanism to grow, Monkewitz and Bunster [7] later concluded that the linear transfer of energy between modes might very well follow points of inflexion. 
With reference to Figure $4.2(\mathrm{~d})$, with $\alpha=14.1, R e_{\delta}=500$, the initial disturbance field is localised near the wall with energy peaks near the linear critical layer and neighbouring inflexion points; we also note that at $\omega t=2 \pi$ the disturbance exists on only one side of the channel. In general, as $R e_{\delta}$ is lowered the frequency of transition decreases, the intensity of the turbulence is less and the rate and extent of propagation into the centre of the channel decreases. These general trends can be seen by comparing Figure $4.2(\mathrm{c})$ and (d), for $\alpha=14.1, R e_{\delta}=800$, and $R e_{\delta}=500$ respectively. Akhavan et al. [10] investigated the primary instability with $\alpha=14.1$ and $R e_{\delta}=1000$ using a spectral method and using a linearised model of the Navier-Stokes equations. The flow was initially disturbed with a wavenumber $\lambda=0.5$ and the evolution of the energy across the width of the channel was plotted. As in the case of the current investigation the initial disturbance field was localised near the wall with energy peaks near the linear critical layer and neighbouring inflexion points with the disturbances quickly migrated toward the centre of the channel.

\subsection{Future Work}

The two dimensional analysis presented here is relevant in three dimensional flows since in the transition regime the primary instability is expected to be two-dimensional. This primary instability does not appear to transfer energy directly, but aids effective stretching of the three-dimensional disturbance, an important part of the secondary mechanism which appears to be responsible for the explosive turbulence observed in experimental flows towards the end of the acceleration phase. Evidently three dimensional modelling is required to investigate this further

The evaluation and application of the LBM in the current investigation serves as a prelude to numerical and experimental studies in vascular applications. Therefore effects such as elastic boundaries and geometrical effects need to be addressed.

\section{Conclusions}

The lattice Boltzmann method has been successfully applied to oscillatory channel flow with a zero mean velocity for a broad range of $\alpha$ and $R e_{\delta}$. In the laminar regime there was good agreement between numerical and exact analytical solutions. The accuracy of the implemented scheme was found to be of first order in space and time. The primary instability was examined and found to be consistent with quasi-steady linear stability theories and previous numerical investigations.

\section{Acknowledgments}

This work was partially supported by EPSRC (UK) under grant GR/N16778 and this assistance is gratefully acknowledged. 
[1] P. Merkli and H. Thomann. Transition to turbulence in oscillating pipe flow. Journal of Fluid Mechanics, 68:567-575, 1975 .

[2] M. Hino, M. Sawamoto, and S. Takasu. Experiments on transition to turbulence in an oscillating pipe flow. Journal of Fluid Mechanics, 75:193-207, 1976.

[3] M. Ohmi, M. Iguchi, K. Kakehachi, and T. Masuda. Transition to turbulence and velocity distribution in an oscillating pipe flow. Bull. JSME, 25:365-371, 1982.

[4] M. Hino, M. Kashiwayanagi, A. Nakayama, and T. Hara. Experiments on the turbulent statistics and the structure of a reciprocating oscillatory flow. Journal of Fluid Mechanics, 131:363-400, 1983 .

[5] M. H. A. Sinada. Inflexion instability and the instability of time-dependent flow. PhD thesis, University of Edinburgh, 1984.

[6] R. Akhavan, R. D. Kamm, and A. H. Shapiro. An investigation of transition to turbulence in bounded oscillatory Stokes flows. Part 1. Experiments. Journal of Fluid Mechanics, 225:395$422,1991$.

[7] P. A. Monkewitz and A. Bunster. The stability of the Stokes layer: visual observations and some theoretical considerations. In Stability of time dependent and spatially varying flows, editors, Dwoyer, D. L. and Hussaini M. Y., pages 244-260, Springer-Verlag, New York, 1987.

[8] S. J. Cowley. High frequency Rayleigh instability of Stokes layers. In Stability of time dependent and spatially varying flows, editors, Dwoyer, D. L. and Hussaini M. Y., pages 261-275, SpringerVerlag, New York, 1987.

[9] C. Von. Kerczek and S. H. Davis. Linear stability theory of oscillatory Stokes layers. Journal of Fluid Mechanics, 62:753-773, 1974.

[10] R. Akhavan, R. D. Kamm, and A. H. Shapiro. An investigation of transition to turbulence in bounded oscillatory Stokes flows. Part 2. Numerical simulations. Journal of Fluid Mechanics, 225:423-444, 1991.

[11] S. Chen and G. D. Doolen. Lattice Boltzmann method for fluid flows. Annual Review of Fluid Mechanics, 30:329-364, 1998.

[12] S. Succi. The Lattice Boltzmann Equation for Fluid Dynamics and Beyond. Oxford University Press, 2001.

[13] F. A. Engelund. Hydrodynamik. Technical University of Denmark, 1968.

[14] Y. H. Qian, D. d'Humieres, and P. Lallemand. Lattice BGK models for Navier-Stokes equation. Europhysics Letters, 17(6):479-484, 1992.

[15] S. Chen, H. Chen, D. Martinez, and W. Matthaeus. Lattice Boltzmann model for simulation of magnetohydrodynamics. Physical Review Letters, 67 (27):3776-3779, 1991.

[16] P. L. Bhatnagar, E. P. Gross, and M. Krook. A model for collision processes in gases. I: small amplitude processes in charged and neutral one-component system. Physical Review, 94:511$525,1954$.

[17] S. Hou, Q. Zou, S. Chen, G. D. Doolen, and A. C. Cogley. Simulation of cavity flow by the lattice Boltzmann equation. Journal of Computational Physics, 118:329-347, 1995.

[18] D. R. Noble, J. G. Georgiadis, and R. O. Buckius. Direct assessment of lattice Boltzmann hydrodynamics and boundary conditions for recirculating flows. Journal of Statistical Physics, $81(1 / 2): 17-33,1995$.

[19] P. A. Skordos. Initial and boundary conditions for the lattice Boltzmann method. Physical Review E, $48(6): 4823-4842,1993$.

[20] D. P. Ziegler. Boundary conditions for lattice Boltzmann simulations. Journal of Statistical Physics, 71 (5/6):1171-1177, 1993.

[21] S. Chen and D. Martinez. On boundary conditions in lattice Boltzmann methods. Physics of Fluids, 8 (9):2527-2536, 1996.

[22] X. He, Q. Zou, L.-S. Luo, and M. Dembo. Analytic solutions of simple flows and analysis of nonslip boundary conditions for the lattice boltzmann bgk model. Journal of Statistical Physics, 87:115$136,1997$. 
[23] S. Succi. The Lattice Boltzmann Equation for Fluid Dynamics and Beyond, page 94. Oxford University Press, 2001.

[24] J. M. Buick and C. A. Greated. Gravity in a lattice Boltzmann model. Phys. Rev. E, 61:5307-5320, 2000 .

[25] D. O. Martinez, W. H. Matthaeus, S. Chen, and D. C Montgomery. Comparison of spectral method and lattice Boltzmann simulations of two- dimensional hydrodynamics. Phys. Fluids, 6(3):1285-1298, 1994.

[26] J. G. M. Eggels. Direct and large-eddy simulations of turbulent fluid flow using the latticeBoltzmann scheme. International Journal of Heat and Fluid Flow, 17:307-323, 1996.

[27] A. M. Artoli, A. G. Hoekstra, and P. M. A. Sloot. Accuracy of 2D pulsatile flow in the lattice Boltzmann BGK method. Springer Lecture Notes in Computer Science, 2329:361-370, 2002.

[28] X. He and L.-S. Luo. Lattice boltzmann model for the incompressible Navier-Stokes equation. Journal of Statistical Physics, 88:927-944, 1997.

[29] M. B. Reider and J. D. Sterling. Accuracy of discrete-velocity BGK models for the simulation of the incompressible Navier-Stokes equations. Computers \& Fluids, 24:459-467, 1995. 


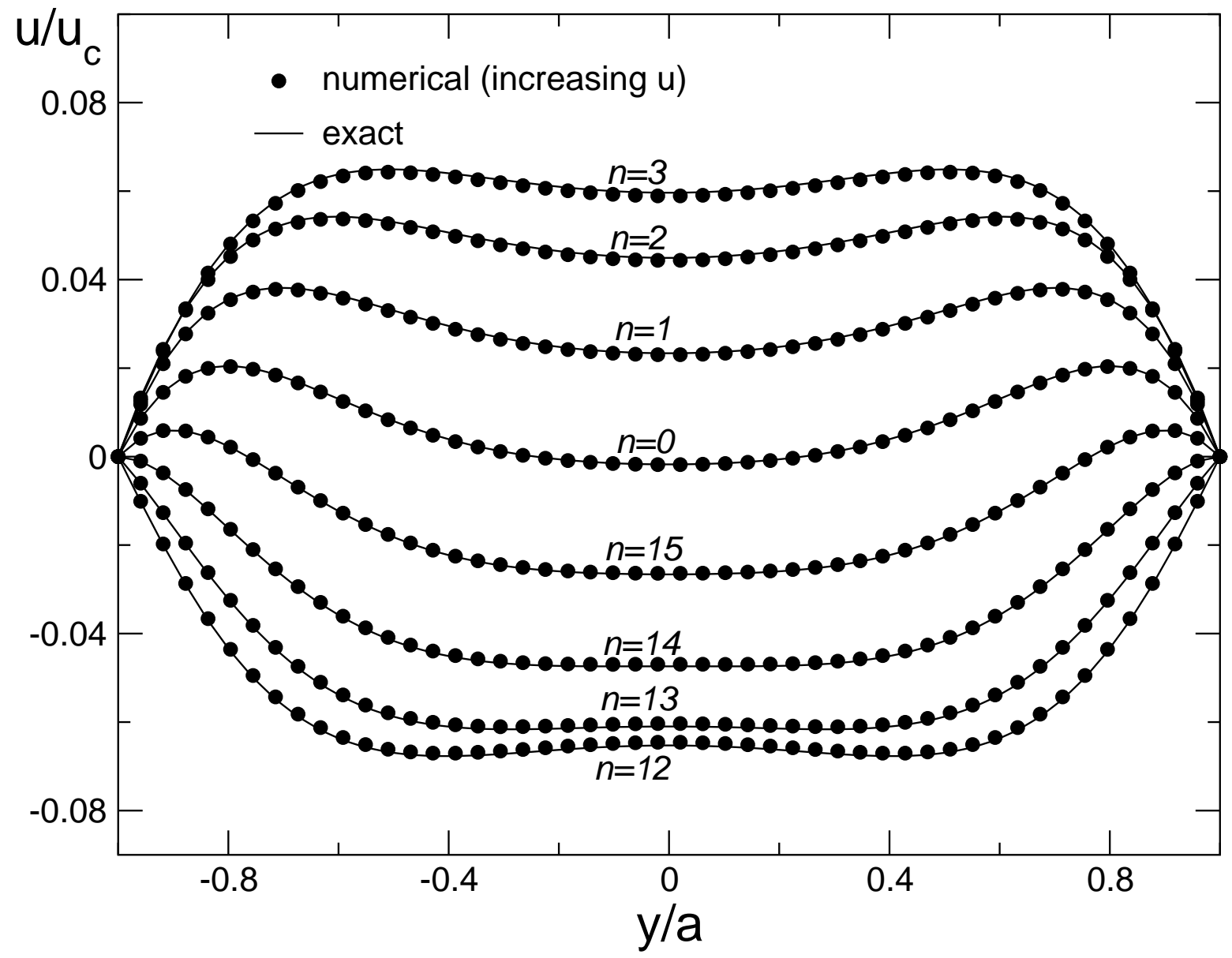

Figure 1a 


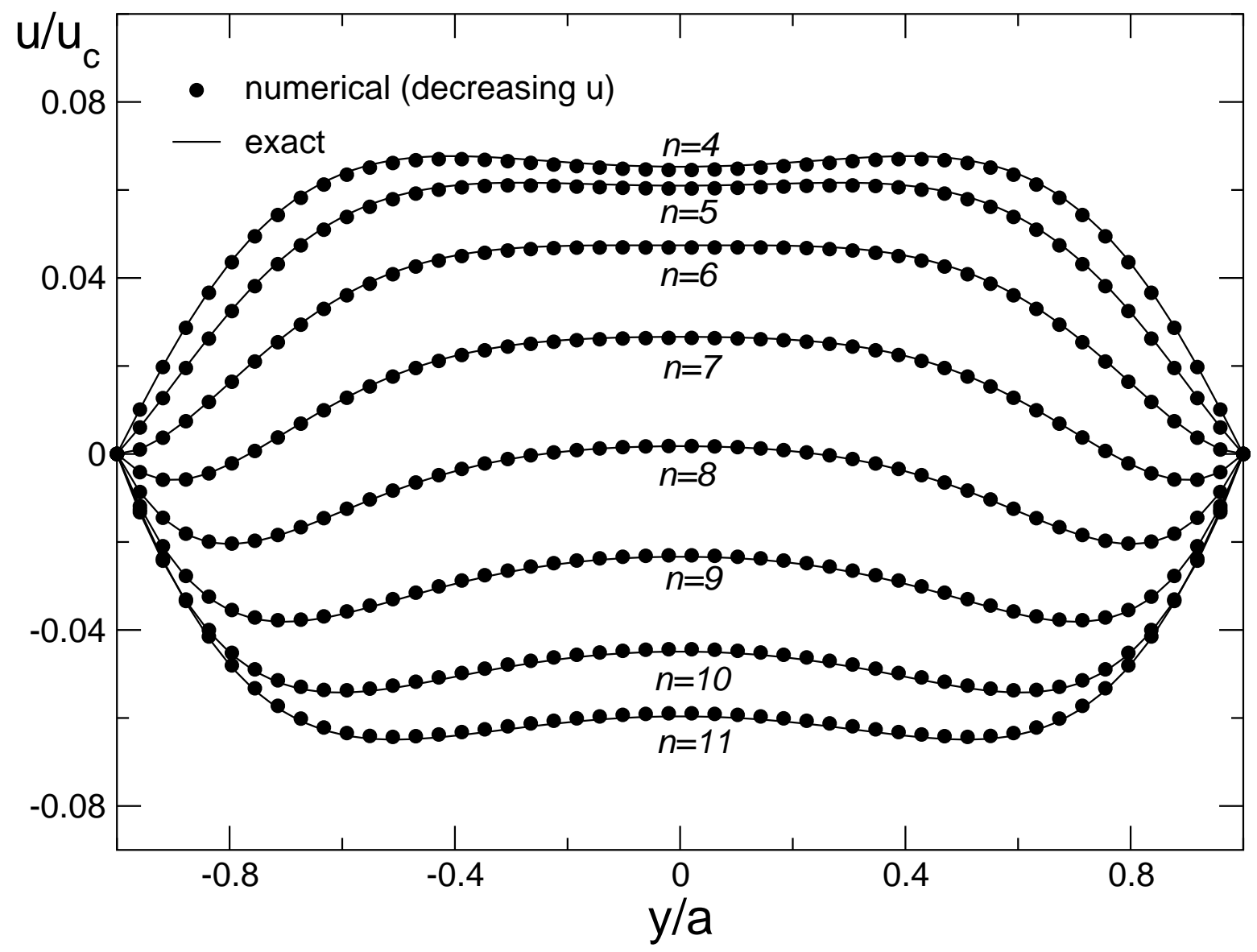

Figure 1b 


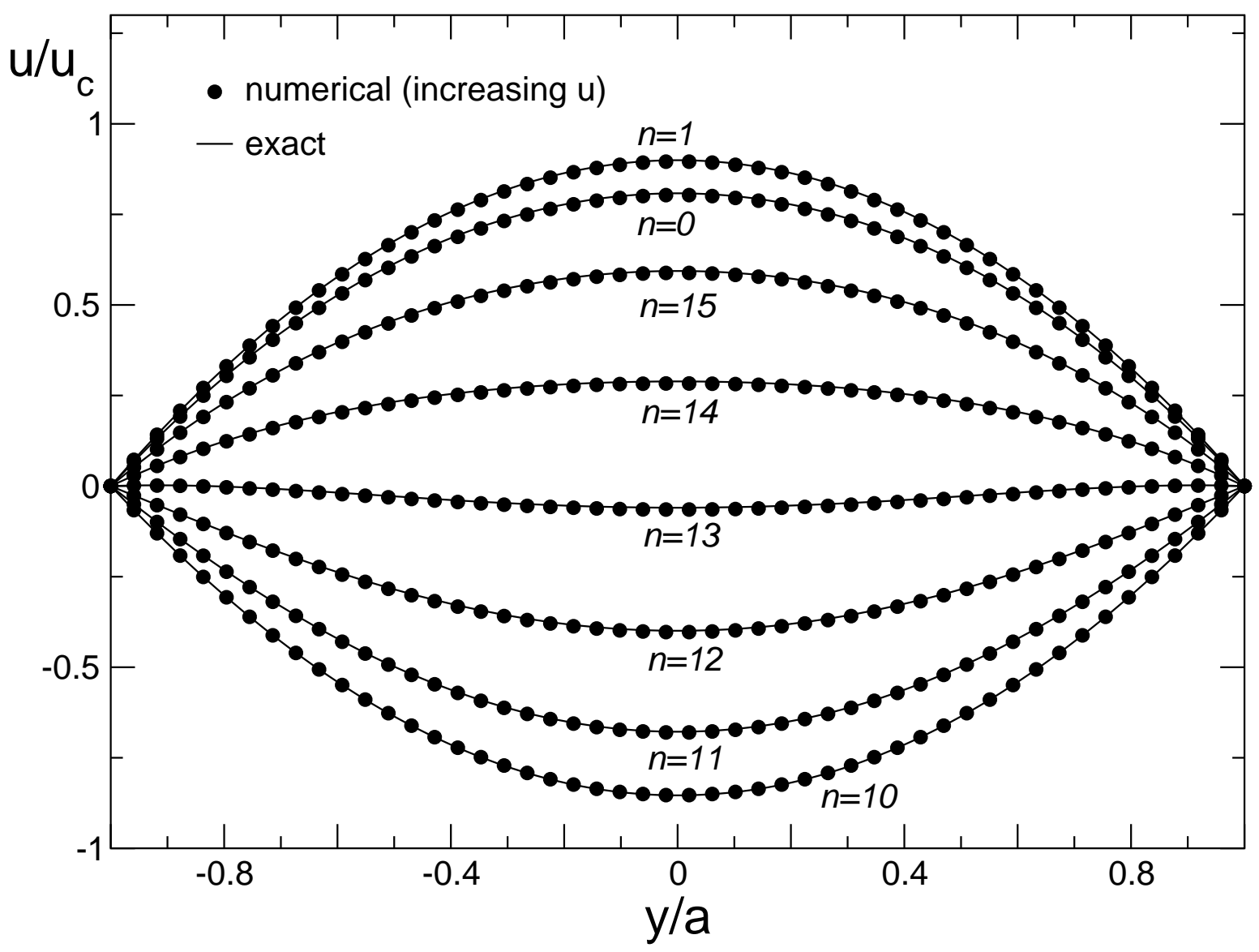

Figure 1c 


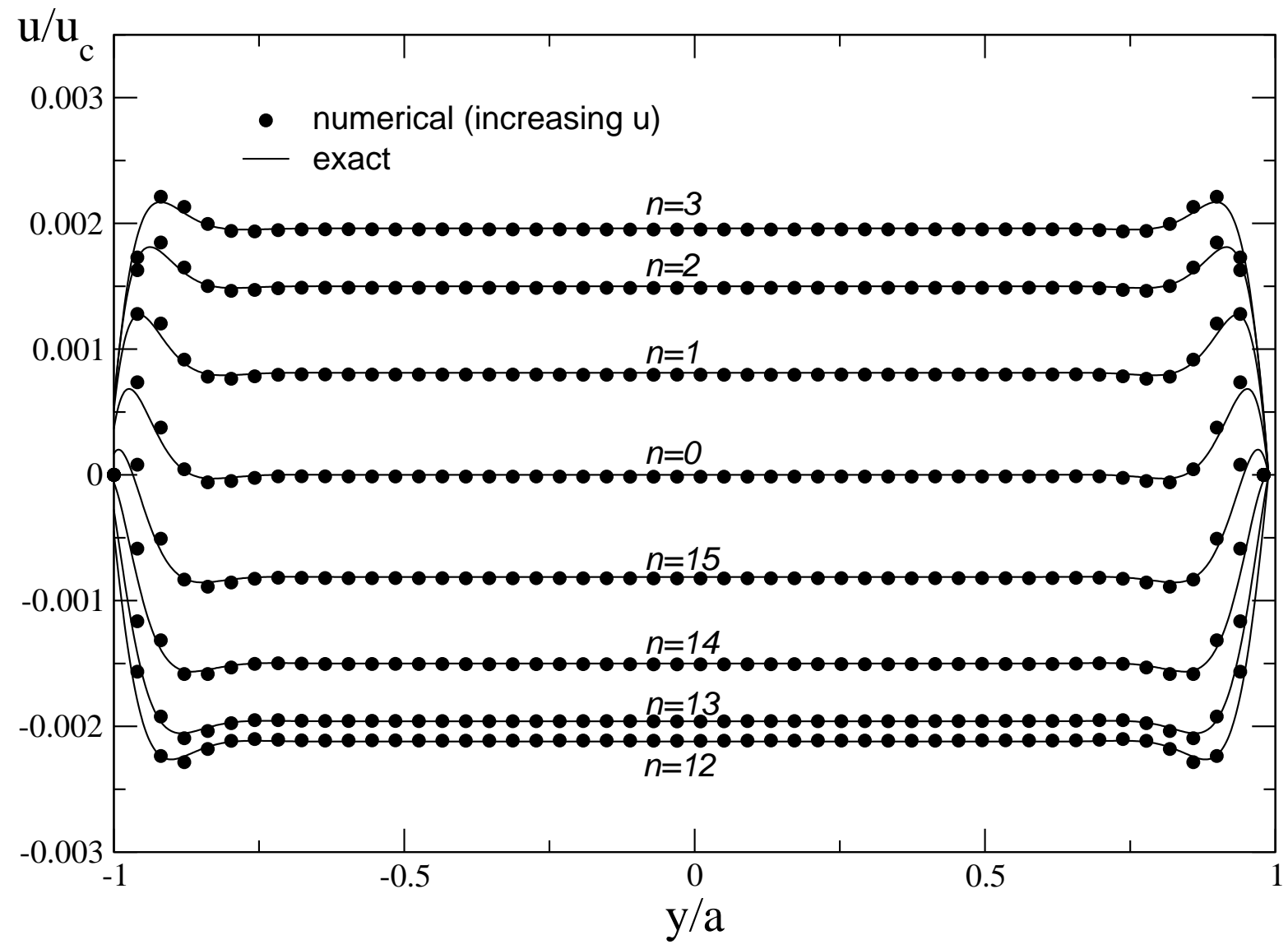

Figure 1d 


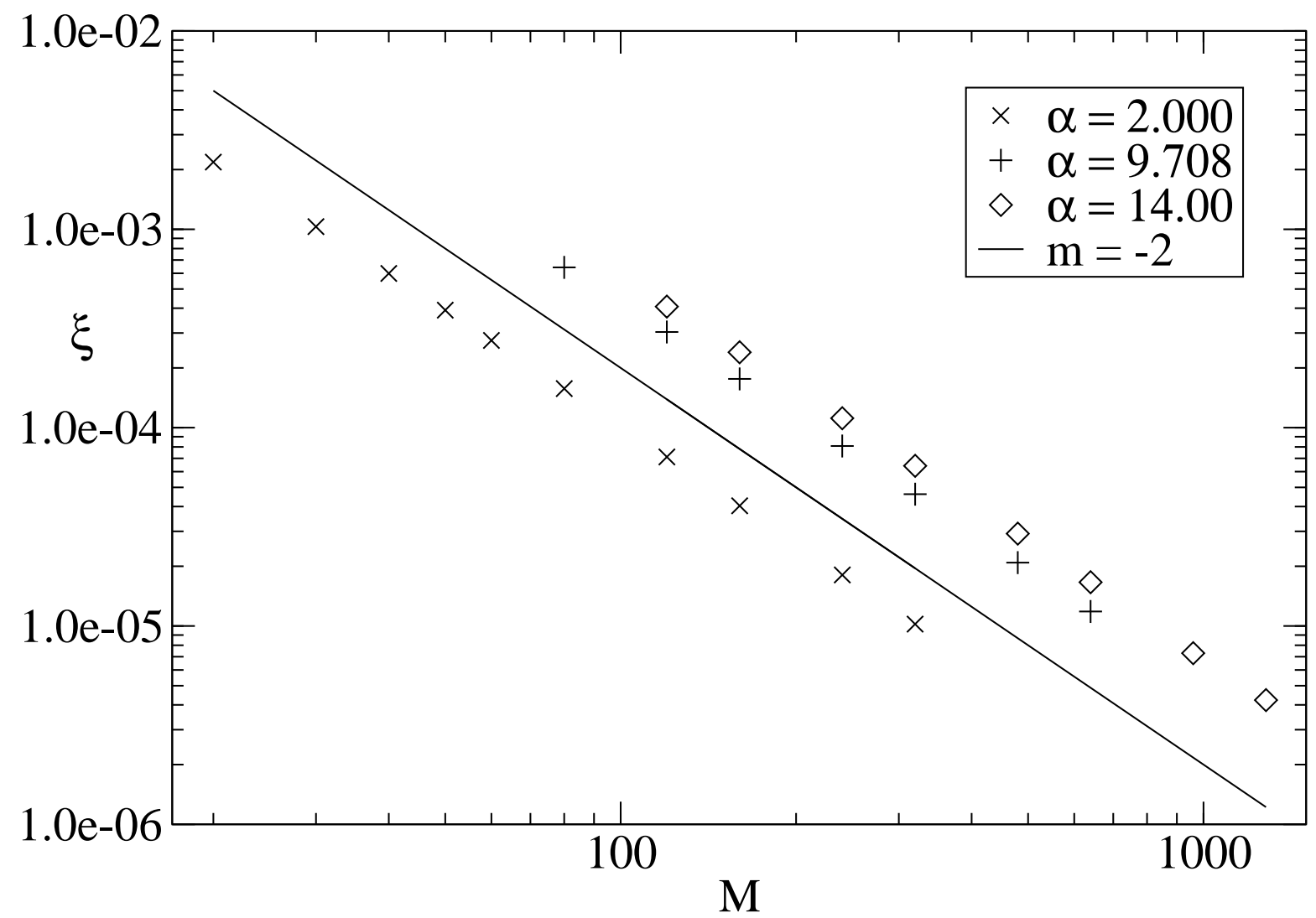

Figure 2 


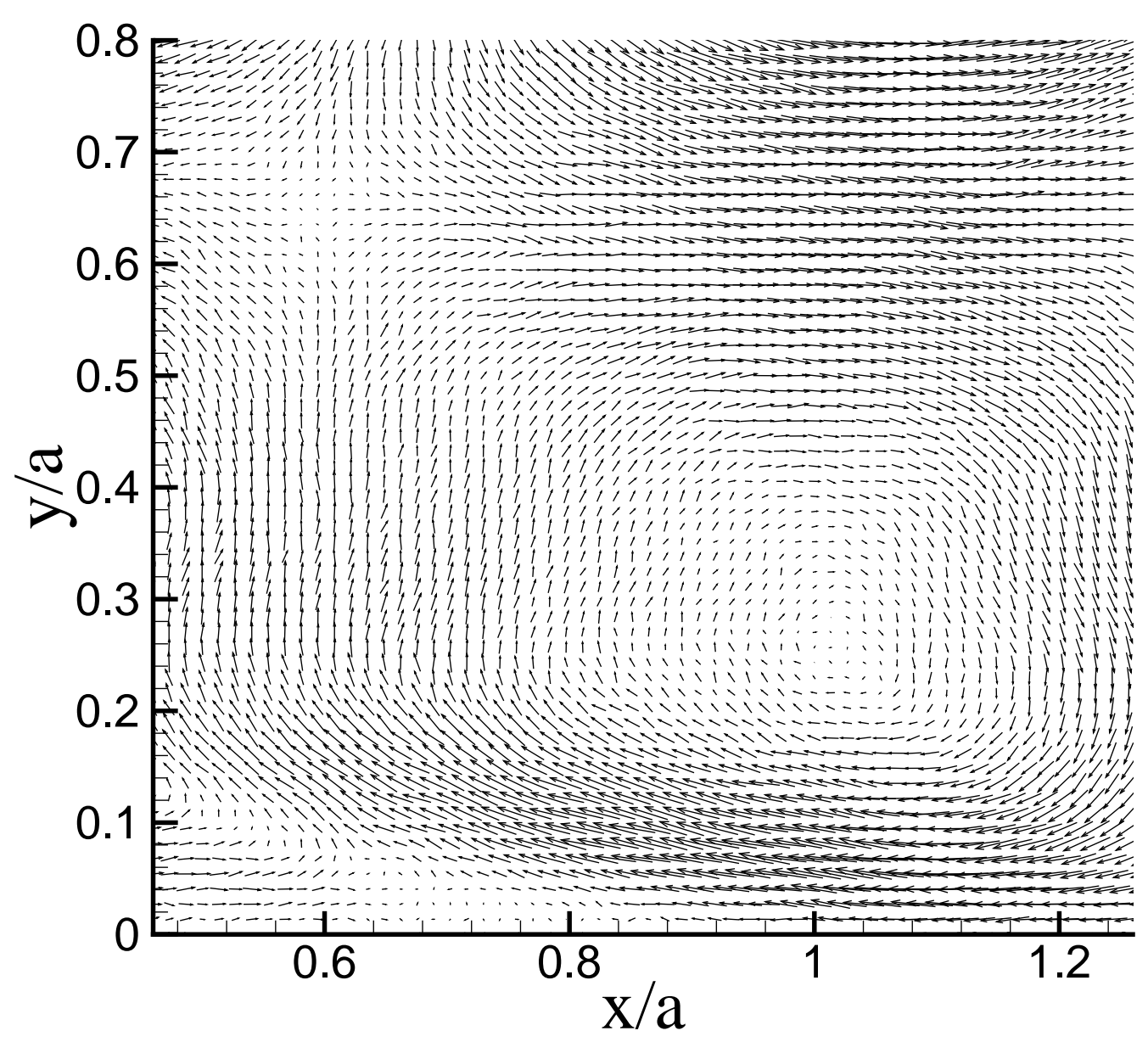

Figure 3 


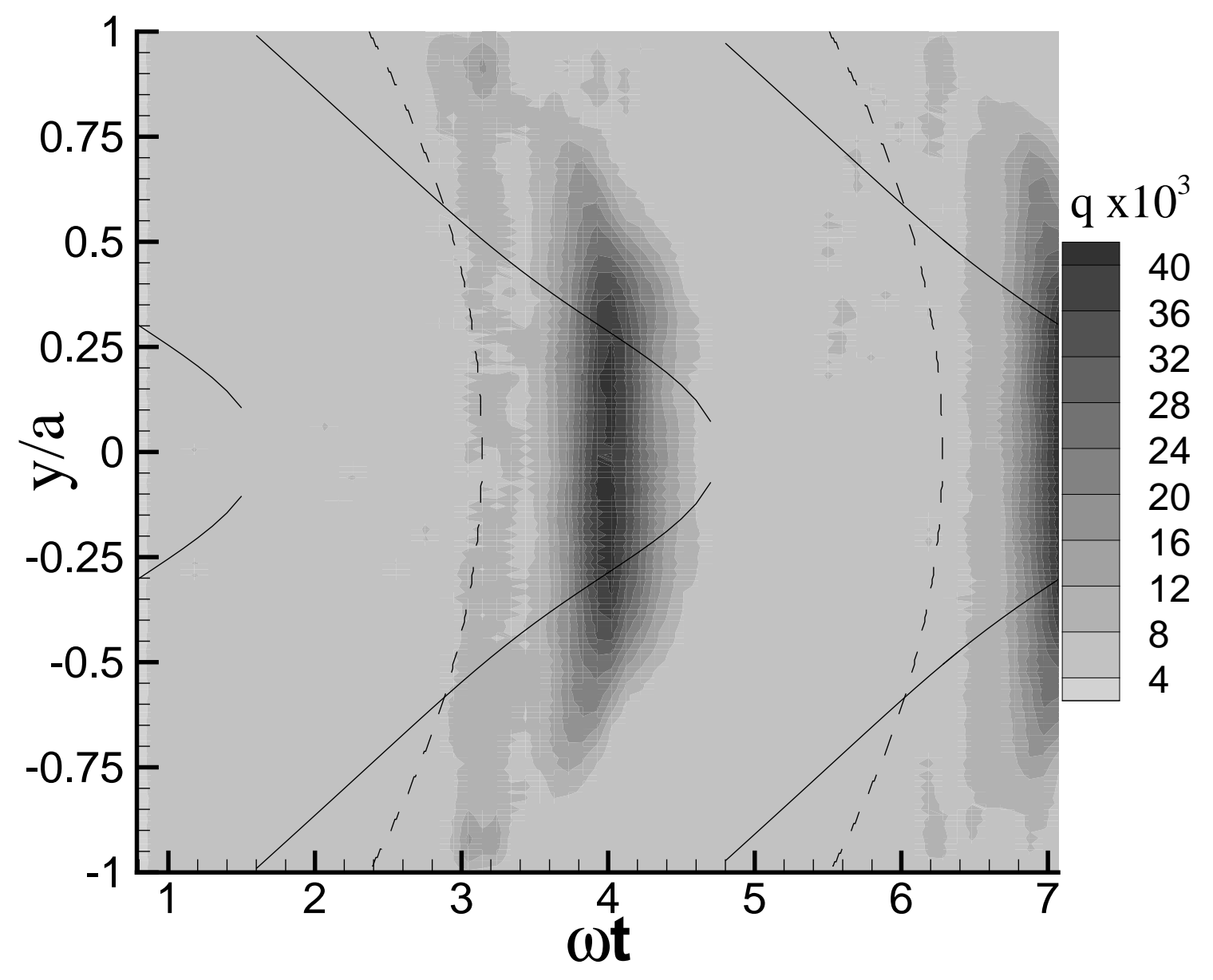

Figure 4a 


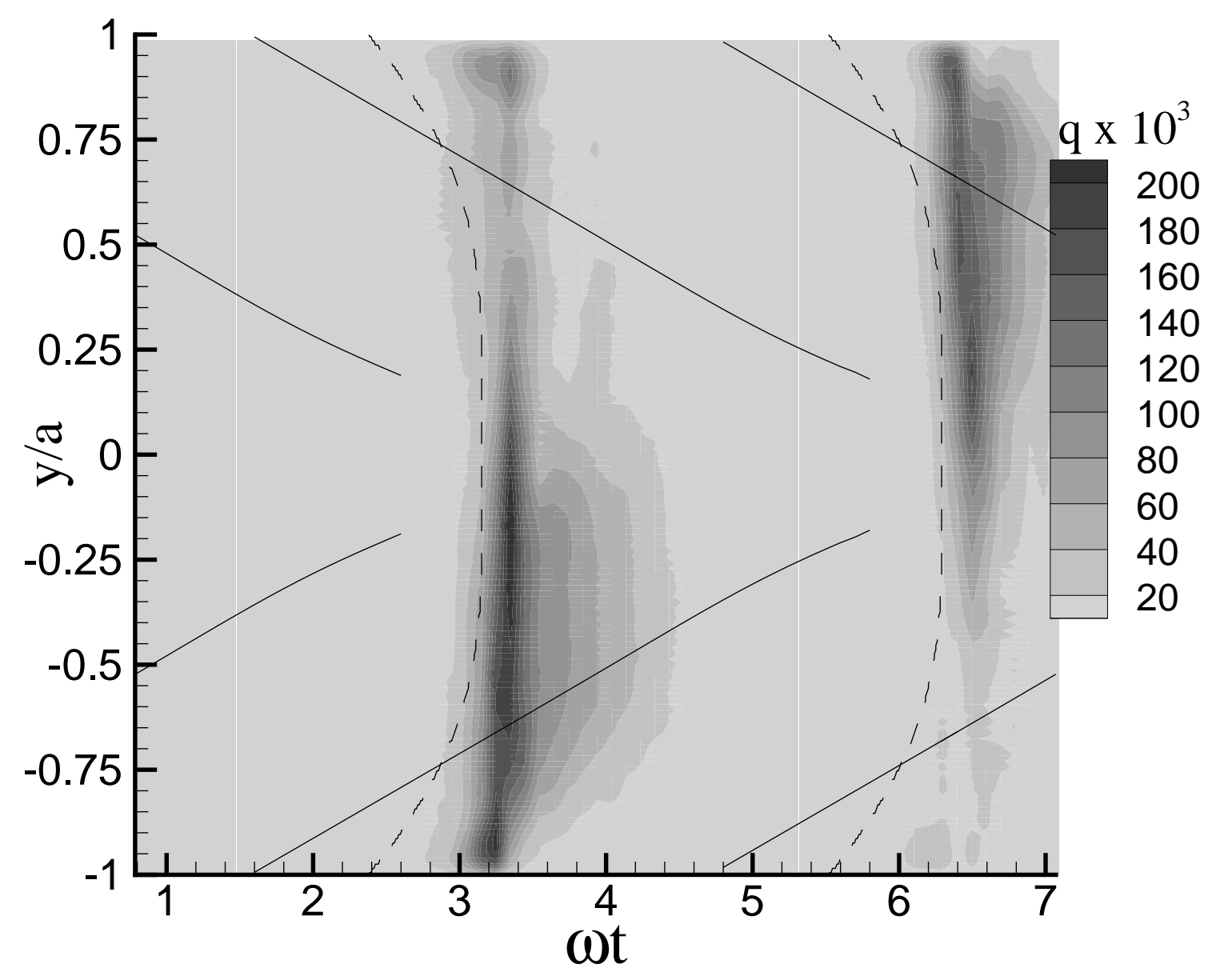

Figure $4 b$ 


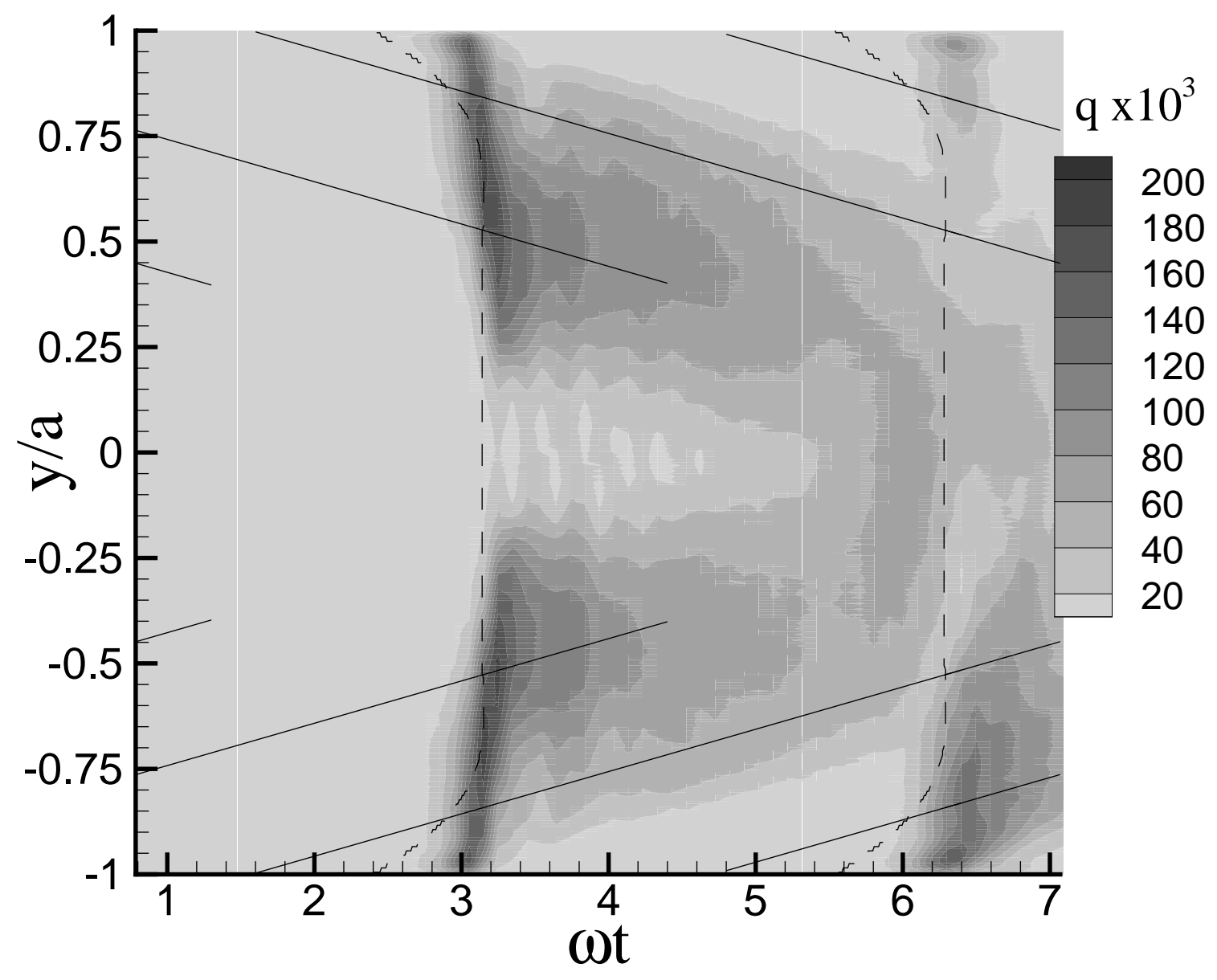

Figure 4c 


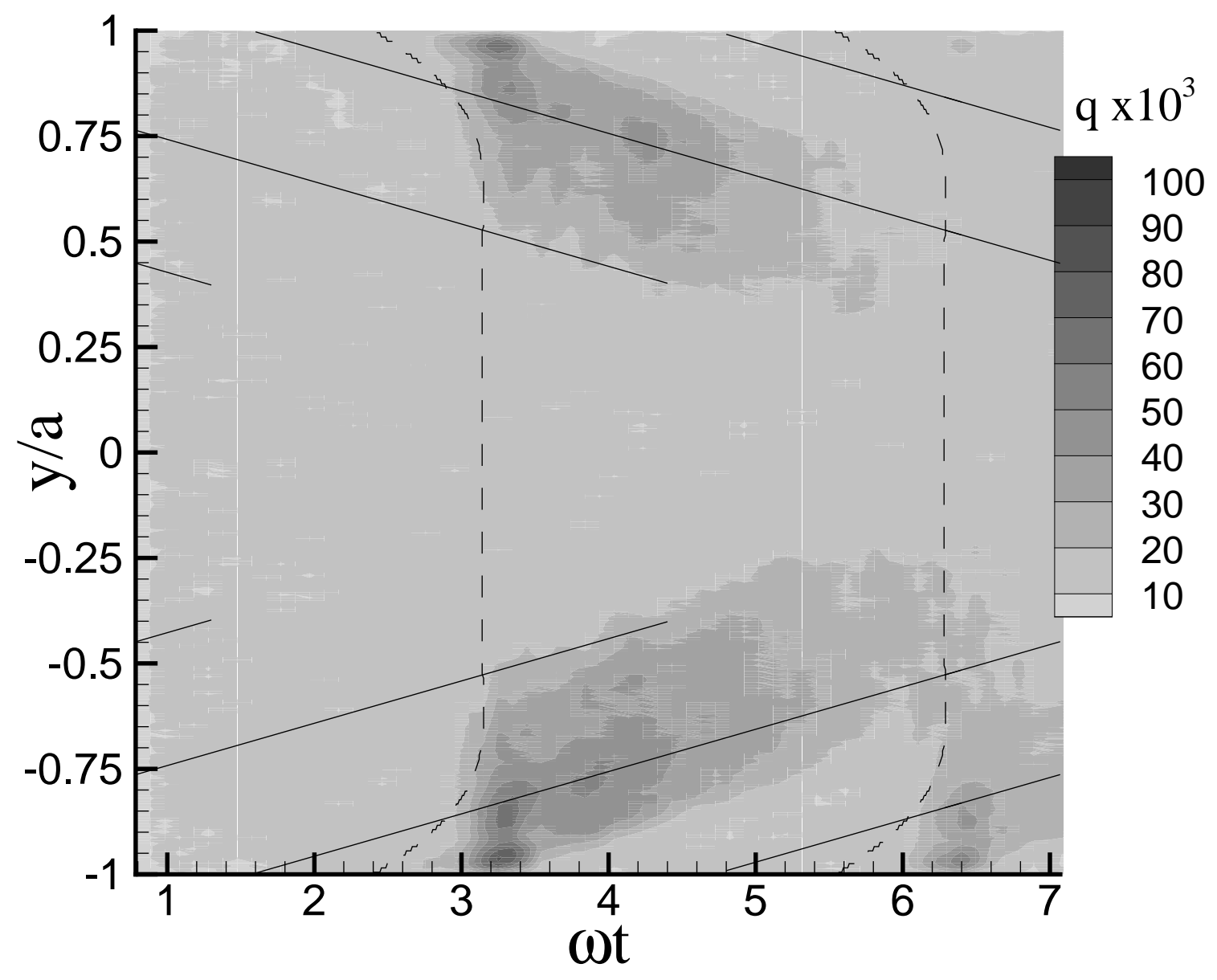

Figure 4d 